\title{
Estudio de corte transversal sobre el estado de conocimientos, actitudes y prácticas de médicos colombianos ante el tabaquismo
}

\author{
A Cross-Sectional Study carried out about the State of knowledge, \\ Attitudes and Practices of Colombian Doctors Facing Smoking
}

\section{Estudo transversal sobre o estado dos conhecimentos, das atitudes $e$ das práticas dos médicos colombianos frente aos fumadores}

\author{
Claudia Ximena Robayo-González, MD. *
}

Juan Carlos Uribe-Caputi, MD., MSc. **

\begin{abstract}
Resumen
Introducción: El consumo de cigarrillo ha venido en aumento entre otras razones por nuevas estrategias comerciales, falta de una política pública definida, contrabando y falta de programas efectivos contra la adicción. Los médicos no son ajenos a este problema con el agravante de que no cuentan con el entrenamiento necesario para influir en la educación de los pacientes. Objetivo: Evaluar los conocimientos, actitudes y prácticas de médicos colombianos respecto al tabaquismo. Metodología: Estudio de corte transversal mediante encuesta virtual realizada a 431 médicos colombianos anónimos, autoseleccionados. Resultados: La prevalencia de vida de consumo de cigarrillo fue del $68.68 \%$. La edad promedio de consumo fue 16 años, el $37.28 \%$ ha fumado más de 5 cajetillas a lo largo de la vida; solamente el $12.54 \%$ de los fumadores ha intentado dejar el hábito; el $61.71 \%$ de los encuestados refirieron uno o ambos padres fumadores,
\end{abstract}

variable que resultó asociada al consumo. El 51.04\% refiere no tener conocimientos para brindar asesoría a los pacientes en el tema; $88.17 \%$ está de acuerdo con la normatividad restrictiva existente en el país; el 95.82\% consideran que son un modelo a seguir y que desempeñan un papel importante frente al paciente. Conclusiones: La prevalencia de tabaquismo activo en médicos colombianos es baja en comparación con la estadística mundial; la asesoría para abandonar el hábito en los pacientes es casi nula. Hay consenso en la necesidad de una mejor formación en el tema entre el personal médico. [Robayo-González CX, UribeCaputi JC. Estudio de corte transversal sobre el estado de conocimientos, actitudes y prácticas de médicos colombianos ante el tabaquismo. MedUNAB 2017-2018; 20(3): 327-337].

Palabras clave: Educación Médica Continua; Cese del Tabaquismo; Uso de Tabaco; Encuestas Epidemiológicas; Hábito de Fumar.

* Médica general, estudiante de especialización de Epidemiologia, Universidad Autónoma de Bucaramanga, Bucaramanga, Santander, Colombia.

** Médico, magíster en Epidemiologia, docente asociado de la Facultad de Medicina de la Universidad de Santander, docente asociado de la Facultad de Medicina de la Universidad Autónoma de Bucaramanga, Bucaramanga, Santander, Colombia.

Correspondencia: Claudia Ximena Robayo González. Carrera 13 No.136-71, Bogotá, Cundinamarca, Colombia. Teléfono: +57 3004492475. E-mail: claudia.ximenarg@gmail.com 


\section{Abstract}

Introduction: Smoking intake has been increasing, among other reasons, due to new commercial strategies, lack of a defined public policy, smuggling and lack of effective programs against addiction. Doctors are not oblivious to this problem with the aggravating factor that they do not have the necessary training to have influence on the education of patients. Objective: To evaluate the knowledge, attitudes and practices of Colombian doctors regarding smoking. Methodology: A cross-sectional study carried out using a virtual survey of 431 unknown and self-selected Colombian doctors. Results: The lifetime prevalence of smoking intake was $68.68 \%$. The average age of consumption was 16 years, $37.28 \%$ of them have smoked more than 5 packages throughout their lives; only $12.54 \%$ of smokers have tried to give up smoking; $61.71 \%$ of respondents reported one or both smoking parents, a variable that was associated with consumption. $51.04 \%$ of them reported not having knowledge to provide advice to patients on the subject; $88.17 \%$ agree with the restrictive regulations existing in the country; $95.82 \%$ of them consider that they are a model to follow and that they play an important role in front of the patient. Conclusions: The prevalence of active smoking in Colombian physicians is low compared to the global statistics; however, the advice to abandon the habit in patients is almost zero. There is a consensus on the need for better training in the subject among medical staff. [RobayoGonzález CX, Uribe-Caputi JC. A Cross-Sectional Study carried out about the State of knowledge, Attitudes and Practices of Colombian Doctors Facing Smoking. MedUNAB 2017-2018; 20(3): 327-337].

Key Words: Education, Medical, Continuing; Smoking Cessation; Tobacco Use; Health Surveys; Smoking.

\section{Introducción}

El consumo de tabaco en forma de cigarrillo se ha convertido en un problema de salud pública, logrando despertar el interés en la generación de políticas y proyectos que permitan disminuir su consumo y las consecuencias para los fumadores activos y pasivos. Por esto, surge la estrategia "Tobacco Free Initiative" (TFI) de la OMS (Organización Mundial de la Salud) (1) que busca fijar las bases para las políticas que se deben implementar en los países miembros por medio del Convenio Marco para el Control del Tabaco (CMCT), en este caso en particular, la consignada en el artículo 12 sobre las medidas educativas contempladas para el personal de la salud (2). Sin embargo, y a pesar de que Colombia figura como país miembro, muchas de las medidas no han podido ser implementadas, en concreto, lo que respecta a la educación del personal de salud, ya que hasta el momento no existe un proyecto de Ley que reglamente la educación en el tema(3).

El consumo de tabaco no sólo causa enfermedad, también aumenta el riesgo de muerte en pacientes con enfermedad coronaria y cáncer (4), situación que genera preocupación

\section{Resumo}

Introdução: O tabagismo aumentou, entre outras razões, devido à novas estratégias comerciais, falta de uma política pública definida, contrabando e falta de programas efetivos contra o vício. Os médicos não são alheios a este problema com o fator agravante de que eles não têm o treinamento necessário para influenciar na educação dos pacientes. Objetivo: Avaliar o conhecimento, atitudes e práticas dos médicos colombianos em relação ao tabagismo. Metodologia: Estudo transversal através de uma pesquisa virtual de 431 médicos colombianos anônimos, autoselecionados. Resultados: A prevalência ao longo da vida do tabagismo foi de $68.68 \%$. A idade média de consumo foi de 16 anos, $37.28 \%$ fumaram mais de 5 pacotes ao longo de suas vidas; apenas $12.54 \%$ dos fumantes tentaram desistir; $61.71 \%$ dos entrevistados relataram que um ou ambos pais fumavão, esta variável foi associada ao consumo. Um 51.04\% afirmaram o desconhecimento dos meios para fornecer aconselhamento aos pacientes sobre o assunto; $88.17 \%$ concordaram com os regulamentos restritivos existentes no país; $95.82 \%$ consideraram que eles são um modelo a seguir e que eles desempenham um papel importante frente do paciente. Conclusões: A prevalência de tabagismo ativo em médicos colombianos é baixa em comparação com as estatísticas globais; o conselho para abandonar o hábito em pacientes é quase nulo. Há consenso sobre a necessidade de uma melhor formação no assunto entre o pessoal médico. [Robayo-González CX, Uribe-Caputi JC. Estudo transversal sobre o estado dos conhecimentos, das atitudes e das práticas dos médicos colombianos frente aos fumadores. MedUNAB 2017-2018; 20(3): 327-337].

Palavras-chave: Educação Médica Continuada; Abandono do Hábito de Fumar; Uso de Tabaco; Inquéritos Epidemiológicos; Hábito de Fumar.

por los problemas de salud y por los elevados costos que puede ocasionarle a los sistemas de salud. Se calcula que el consumo de tabaco causa cada año más de 7 millones de muertes en el mundo, más que las muertes por el Virus de Inmunodeficiencia Humana (VIH), malaria y tuberculosis (5). De persistir las tendencias actuales, para el siglo 21 , causará más de 1 billón de muertes en el mundo(4), el 70\% de esas muertes prematuras ocurrirán en los países de medianos y bajos ingresos(6).

Como parte de la TFI se desarrollaron una serie de encuestas para adultos, jóvenes y personal de la salud, que buscan evaluar la prevalencia de tabaquismo, así como los comportamientos y el conocimiento de todas las personas; se realizó la prueba piloto en el año 2005 con 10 países, demostrando que la prevalencia de consumo de tabaco en estudiantes de áreas de la salud se encuentra entre el 18.1\% y el $47.1 \%$, y que cerca del $90 \%$ opinan que se debe asesorar a los pacientes, pero solo reciben entrenamiento entre un 5.2\% y un $20.9 \%$ en algunos países(7).

En la encuesta nacional para profesionales de la salud en Estados Unidos sobre la prevalencia de consumo de tabaco, 
prácticas para dejar el uso y creencias, se evidenció que la prevalencia del consumo de cigarrillo es más alto en enfermeras con un $13 \%(8)$. Entre el $87.3 \%$ y el $99.5 \%$ de los médicos siempre indagan sobre su consumo, mientras que entre un $65.6 \%$ y un $94.9 \%$, dan consejo al paciente pero no continúan con los últimos tres pasos de la estrategia $5 \mathrm{As}$ (Ask, Advise, Assess, Assist, Arrange)(8). Esta estrategia se basa en averiguar o preguntar al paciente si fuma, aconsejar abandonar el hábito, apreciar o evaluar la voluntad del paciente para abandonar el consumo de tabaco, ayudar a lograr el abandono por medio de terapias establecidas; y por último arreglar una próxima cita que solo se centre en la consulta para dejar el hábito de fumar. También reportó las creencias por parte de los médicos al momento de asesorar, aspectos como la falta de tiempo, el tipo de servicio en el que trabajan y la incomodidad que perciben al preguntar sobre el hábito de fumar(8).

En Bosnia y Herzegovina, se evaluó a los profesionales de la salud frente al consumo de tabaco, quienes revelaron $45 \%$ de prevalencia de consumo de cigarrillo, sin contar $17 \%$ que alguna vez fumó; la mayoría fumadores por casi 14 años, con un promedio de consumo de 16 cigarrillos por día. También se encontró que cerca del $50 \%$ habían recibido entrenamiento en las técnicas para el cese del hábito, pero sólo el 36\% se sentía preparado para brindar asesoría (9). Sin embargo, se destaca la diferencia con datos adquiridos en encuestas en otros países europeos donde la prevalencia de personal de salud fumador en algunos casos no pasa del 30\%(9).

En Colombia se llevó a cabo una encuesta en el año 2001 en estudiantes de quinto año de medicina, que demostró que el $25.7 \%$ eran fumadores, de los cuales el $91.3 \%$ había tenido deseos de cesar el hábito; el 92.7\% de los encuestados estaban de acuerdo con la política de no fumar en instituciones de salud, pero solo el $63.9 \%$ de los no fumadores y el $38.9 \%$ de los fumadores estaban de acuerdo con la prohibición de fumar en lugares públicos; sin embargo, no se evaluaron los conocimientos respecto a las estrategias para dejar de fumar (10). El objetivo de este estudio es estimar la prevalencia de consumo de cigarrillo, y determinar los conocimientos y actitudes respecto al consumo de tabaco en médicos colombianos.

\section{Metodología}

Se realizó un estudio descriptivo de corte transversal mediante la aplicación de una encuesta basada en la forma de Global Health Professions Student Survey(GHPSS)(11) modificada en español, la cual ha sido utilizada en más de 10 países a nivel mundial incluyendo Argentina, Chile y Perú. Requiere aproximadamente un tiempo de 7 minutos para su diligenciamiento completo.

La encuesta consta de 6 secciones con un total de 42 preguntas: la primera sección consta de 9 preguntas de opción múltiple con única respuesta donde se evalúa la prevalencia de uso de tabaco. La segunda sección consta de 4 preguntas de opción múltiple con única respuesta donde se evalúa la exposición al humo de tabaco ambiental. La tercera sección consta de 11 preguntas de "si" o "no" donde se evalúa actitudes sobre el uso de tabaco en lugares abiertos y cerrados, al igual que en el sitio de trabajo. La cuarta consta de 8 preguntas de selección múltiple sobre conocimientos acerca de comportamientos y cesación del tabaquismo. La quinta sección consta de 7 preguntas de "si" o "no" que indagan acerca del currículo y entrenamiento recibidos sobre el tabaquismo. La sexta y última sección consta de 3 preguntas demográficas.

Los criterios de inclusión fueron: ser médico graduado, estar ejerciendo profesionalmente en Colombia. Como criterio de exclusión tener más de un $20 \%$ de datos perdidos en la encuesta.

La encuesta se envió vía correo electrónico a la Asociación de Médicos Internos y Residentes (ANIR), Federación Médica Colombiana, Sociedad Colombiana de Pediatría y la Federación Colombiana de Ginecología y Obstetricia por medio de la plataforma Surveymonkey.com, la cual permitía la realización de la encuesta desde equipos portátiles, tabletas o teléfonos móviles con sistema Android. Los datos se analizaron en Epi Info 7.1.5. Los datos fueron expresados en cuadros de frecuencias; en cuadros de doble entrada por factores asociados a consumo, razones de prevalencia y Odds Ratio(OR).

De acuerdo con la Resolución 8430 de 1993, del Ministerio de Salud de Colombia, este estudio cumple con los criterios de investigación en salud y por lo tanto está sujeta a las normas éticas correspondientes, sin embargo, no requirió aprobación del Comité de Ética de la facultad de medicina de la Universidad Autónoma de Bucaramanga ya que dicho protocolo no constituye riesgo para los participantes. No se tomaron datos de identificación, ni se revelaron los datos específicos de IP (Internet Protocol) de los participantes.

\section{Resultados}

Se recolectaron 537 registros; por criterios de inclusión se eliminaron 37 por datos demográficos incompletos o ausentes, 33 con más del $20 \%$ de datos perdidos (8 preguntas o menos sin respuesta), 36 que corresponden a otros trabajadores de la salud, dejando un total de 431 encuestas para el análisis. Las características demográficas y el área de trabajo se pueden observar en la tabla 1 (Tabla 1).

Al indagar sobre la historia personal de consumo de cigarrillo, se encontró una prevalencia de vida del $68.68 \%$ (IC95\% 64.03-72.99) ( $\mathrm{n}=296)$, siendo la edad promedio para la experimentación los 16 años; de éstos el 37.76\% (IC 95\% 32.19-43.17) $(\mathrm{n}=111)$ han consumido más de 5 
Tabla 1. Características demográficas de la muestra

\begin{tabular}{lcc}
\hline \multicolumn{3}{c}{ Datos demográficos } \\
\hline Rango de edad & 20 -81 años & 33 años (media) \\
Sexo (n=431) & & \\
Femenino & 242 & $56.15 \%$ \\
Masculino & 189 & $43.85 \%$ \\
Médico (n=431) & & \\
General & 241 & $55.92 \%$ \\
Especialista & 190 & $44.08 \%$ \\
Área de trabajo (n=399) & & \\
Consulta externa & 144 & $36.09 \%$ \\
Dos o más áreas & 125 & $31.33 \%$ \\
Urgencias & 65 & $16.29 \%$ \\
Cirugía & 16 & $4.01 \%$ \\
Administrativo & 12 & $3.01 \%$ \\
Docencia & 12 & $3.01 \%$ \\
Hospitalización & 14 & $3.51 \%$ \\
Consulta privada & 3 & $0.75 \%$ \\
UCl & 3 & $0.75 \%$ \\
Consulta domiciliaria & 2 & $0.50 \%$ \\
Investigación & 2 & $0.50 \%$ \\
Unidad renal & 1 & $0.25 \%$ \\
\hline
\end{tabular}

*UCl: Unidad de Cuidados Intensivos cajetillas durante toda su vida, como se muestra en las tablas 2 y 3 (Tabla 2, Tabla 3).

Se evaluó si durante el último año han existido intentos para dejar de fumar, encontrando que el 24.05\% $(n=71)$ de los médicos han continuado con el hábito pero solo el $12.54 \%$ (IC 95\% 8.99-16.87) (n=9) han intentado dejar de fumar; el lugar donde suelen fumar con mayor frecuencia son los eventos sociales con un $44.62 \%$ de las veces (IC 95\% 32.2757.47) $(\mathrm{n}=29)$ (Tabla 4) y el $31.94 \%$ de los fumadores actuales (IC 95\% 5.47-22.82) $(\mathrm{n}=23)$, ha consumido cigarrillos en su lugar de trabajo durante el último año.

Los 72 médicos que manifestaron consumo en el último año, 44 lo han hecho en el último mes y de estos el $13.89 \%$ (IC 95\% 6.87-24.06) $(\mathrm{n}=10)$ lo ha hecho en promedio durante 9 días y el 19.44\% (IC 95\% 11.06-30.47) $(n=14)$, todos los días (Figura 1).

Tabla 2. Prevalencia de consumo de cigarrillo

\begin{tabular}{cccc}
\hline $\begin{array}{c}\text { Consumo de } \\
\text { cigarrillo }\end{array}$ & $\begin{array}{c}\text { Frecuencia } \\
\text { absoluta }\end{array}$ & Porcentaje & IC 95\% \\
\hline Si & 296 & $68.68 \%$ & $64.03-72.99$ \\
No & 135 & $31.32 \%$ & $27.01-35.97$ \\
Total & 431 & $100.00 \%$ & \\
\hline
\end{tabular}

Tabla 3. Cantidad de cigarrillos consumidos durante su vida.

\begin{tabular}{lccc}
\hline Cantidad de Cigarrillos & Frecuencia absoluta & Porcentaje & IC 95\% \\
\hline 1 cigarrillo & 65 & $22.11 \%$ & $17.50-27.29$ \\
2 a 10 cigarrillos & 84 & $28.57 \%$ & $23.48-34.10$ \\
1 a 5 cajetillas & 34 & $11.56 \%$ & $8.14-15.78$ \\
Más de 5 cajetillas & 111 & $37.76 \%$ & $32.19-43.57$ \\
Total & 294 & $100.00 \%$ & \\
\hline
\end{tabular}

Tabla 4. Lugar donde fuma con mayor frecuencia

\begin{tabular}{lccc}
\hline Lugar en donde fuma & Frecuencia absoluta & Porcentaje & IC 95\% \\
\hline Eventos sociales & 29 & $44.62 \%$ & $32.27-57.47$ \\
Espacios públicos & 14 & $21.54 \%$ & $12.31-33.49$ \\
Casa & 10 & $15.38 \%$ & $7.63-26.48$ \\
Lugar de trabajo & 8 & $12.31 \%$ & $5.47-22.82$ \\
Casa de amigos & 4 & $6.15 \%$ & $1.70-15.01$ \\
Total & 65 & $100.00 \%$ & \\
\hline
\end{tabular}




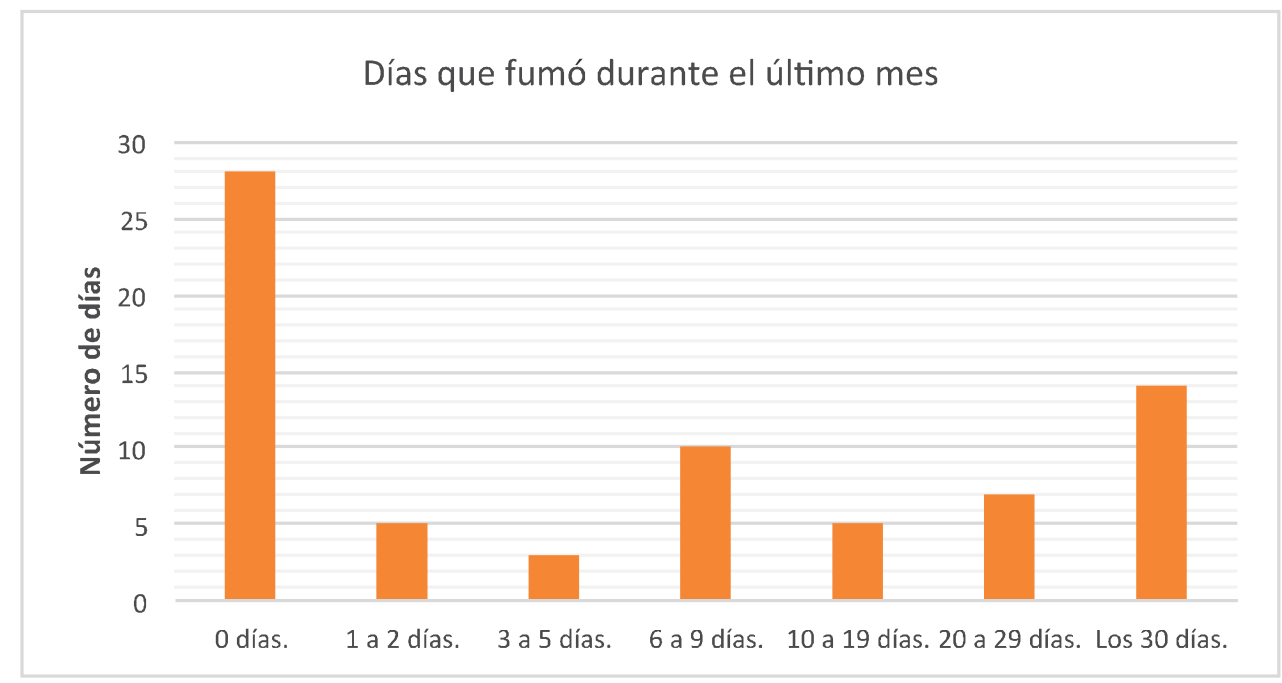

Figura 1. Días que fumó en el último mes.

Se evaluó el grado de dependencia al cigarrillo encontrándose que el $20.45 \%$ de los encuestados (IC $95 \%$ 9.8-35.3) (n=9) sienten deseos de fumar a los 60 minutos de despertar; 28 profesionales fumadores afirmaron su deseo de abandonar el consumo y solamente 9 han recibido ayuda para abandonar el hábito de fumar, sin encontrarse asociación en estas variables.

La exposición al cigarrillo por parte de los padres, mostró asociación entre probar el cigarrillos y la presencia de uno o ambos padres fumadores OR 1.9 (IC 95\% 1.2-2.8 $\mathrm{p}=0.002$ ) (Tabla 5). El $91.42 \%(n=394)$ y el $44.84 \%(n=191)$ de los encuestados no ha tenido exposición a humo de segunda mano en el hogar ni en algún lugar diferente respectivamente; sin embargo, el $55.16 \%(\mathrm{n}=235)$ ha estado expuesto al humo de segunda mano por lo menos por una persona en la semana (Tabla 6).

Con respecto al tema de reglamentación sobre el consumo de cigarrillo, 44 profesionales aseguran que no existe una

Tabla 5. Exposición al cigarrillo por parte de los padres

\begin{tabular}{lccc}
\hline Padres fumadores & Frecuencia absoluta & Porcentaje & IC 95\% \\
\hline Ambos & 75 & $17.40 \%$ & $14.01-21.39$ \\
Madre & 52 & $12.06 \%$ & $9.22-15.61$ \\
Padre & 139 & $32.25 \%$ & $27.90-36.92$ \\
Ninguno & 160 & $37.12 \%$ & $32.58-41.90$ \\
No sabe & 5 & $1.16 \%$ & $0.43-2.85$ \\
Total & 431 & $100.00 \%$ & \\
\hline
\end{tabular}

Tabla 6. Exposición a humo de segunda mano fuera de la vivienda

\begin{tabular}{lccc}
\hline En los últimos 7 días ¿cuántas personas han fumado en su presencia en un lugar diferente a donde vive? \\
\hline Número de personas & Frecuencia & Porcentaje & IC $95 \%$ \\
0 personas. & 191 & $44.32 \%$ & $39.58-49.15$ \\
1 a 2 personas. & 151 & $35.03 \%$ & $30.57-39.77$ \\
3 a 4 personas. & 53 & $12.30 \%$ & $9.42-15.86$ \\
5 a 6 personas. & 17 & $3.94 \%$ & $2.39-6.37$ \\
7 personas. & 14 & $3.25 \%$ & $1.86-5.52$ \\
Datos perdidos & 5 & $1.16 \%$ & $0.43-2.85$ \\
Total & 431 & $100.00 \%$ & \\
\hline
\end{tabular}


política oficial que prohíba el consumo en lugares de trabajo o la desconocen (10.21\%); de otro lado, el 6.28\% $(\mathrm{n}=27$; IC 95\% 4.26-9.12) afirmó que la política no se cumple (Tabla 7).

En relación a la evaluación de los conocimientos del médico para asesorar al paciente, los médicos aseguran que se ha discutido durante su carrera los peligros y consecuencias del cigarrillo, sobre la importancia del registro de esta información en la historia clínica y de proveer de material de apoyo a los pacientes con consumo de cigarrillo; sin embargo, el 34.57\% (IC 95\% 30.12-39.30) (n=149) no han tenido sesiones donde se discuta los motivos por los cuales las personas fuman y el $51.04 \%(\mathrm{n}=220)$ de los encuestados asegura no tener el conocimiento suficiente para asesorar a estos pacientes (Tabla 8). Cabe aclarar que estas preguntas solo evalúan el conocimiento más no la aplicación a los pacientes con consumo de tabaco.

La opinión acerca de las políticas de prohibición reveló que la mayoría está de acuerdo con no permitir la venta a menores de edad, la prohibición de todo tipo de publicidad del cigarrillo y la prohibición de fumar en discotecas, restaurantes y lugares públicos (Tabla 9). De los fumadores que han intentado dejar el hábito en el último año, el 97.30\% están de acuerdo con la necesidad de la prohibición en restaurantes, $78.38 \%$, en lugares públicos y $86.49 \%$ en bares.

Tabla 7. Información sobre la política de prohibición

\begin{tabular}{lcc}
\hline \multicolumn{4}{l}{ ¿Sabe usted de una política oficial que prohíba fumar en su lugar de trabajo? } \\
\hline & $\begin{array}{c}\text { Frecuencia } \\
\text { absoluta }(n=431)\end{array}$ & Porcentaje \\
No hay una política oficial & 15 & $3.48 \%$ \\
No sabe & 29 & $6.73 \%$ \\
Si, para cualquier lugar público abierto & 28 & $6.50 \%$ \\
Si, para cualquier lugar público cerrado & 326 & $75.64 \%$ \\
Si, para las clínicas solamente & 23 & $5.34 \%$ \\
Si, para las instalaciones de las universidades solamente & 10 & $2.32 \%$ \\
\hline ¿Se cumple la política de prohibición de fumar en su lugar de trabajo? & \\
\hline \multicolumn{4}{c}{ Frecuencia } \\
No sabe & absoluta ( $\mathrm{n}=430)$ & \\
No, la política no es cumplida & 35 & $8.14 \%$ \\
Si, la política es cumplida & 27 & $6.28 \%$ \\
\hline
\end{tabular}

Tabla 8. Conocimientos sobre tabaquismo

\begin{tabular}{|c|c|c|}
\hline Conocimientos & & \\
\hline & Si & No \\
\hline $\begin{array}{l}\text { Durante su carrera protesional ¿le han hablado en alguna de sus clases sobre los peligros de } \\
\text { fumar? }\end{array}$ & $422(98.14 \%)$ & $8(1.86 \%)$ \\
\hline $\begin{array}{l}\text { Durante su carrera ¿en algunas de sus clases se ha discutido acerca de las razones de } \\
\text { porque la gente fuma? } \\
\text { Ha oído hablar de la utilización de terapias de reemplazo de nicotina en los programas para }\end{array}$ & $278(65.11 \%)$ & $149(34.89 \%)$ \\
\hline $\begin{array}{l}\text { dejar de fumar? (como parches o goma de mascar) } \\
\text { ¿Ha oído hablar acerca del uso de los antidepresivos en los programas para dejar de fumar? }\end{array}$ & $416(96.97 \%)$ & $13(3.03 \%)$ \\
\hline (como el bupropion o zyban, champix) & $260(60.32 \%)$ & $171(39.68 \%)$ \\
\hline $\begin{array}{l}\text { Durante su carrera profesional ¿alguna vez ha aprendido acerca de la importancia de registrar } \\
\text { en la historia clínica el consumo de tabaco como parte general de la historia médica? }\end{array}$ & $424(98.60 \%)$ & $6(1.40 \%)$ \\
\hline $\begin{array}{l}\text { Durante su carrera profesional ¿usted ha recibido alguna formación acerca de cómo dejar de } \\
\text { fumar y cómo aplicar esto en los pacientes? }\end{array}$ & $210(48.84 \%)$ & $220(51.16 \%)$ \\
\hline $\begin{array}{l}\text { Durante su carrera profesional ¿usted ha aprendido la importancia de proveer material } \\
\text { educativo y de apoyo a los pacientes que quieren dejar de fumar? }\end{array}$ & $277(64.72 \%)$ & $151(35.28 \%)$ \\
\hline
\end{tabular}


En cuanto a la importancia del rol como médicos frente a los pacientes y el público en general, el $86.51 \%$ considera que los profesionales de la salud ejercen un rol de modelo para los pacientes y el público en general y el $95.82 \%$ cree que los profesionales de la salud tienen algún rol en aconsejar o dar información a sus pacientes acerca de cómo dejar de fumar. El 90.49\% (IC 95\% 87.22-93.01) ( $\mathrm{n}=390)$ considera que los profesionales de la salud deben recomendar a los pacientes de forma rutinaria los métodos para dejar de fumar mientras que el $34.57 \%$ (IC 95\% 30.12-39.30) $(n=149)$, creen que se debería recomendar a los pacientes de forma rutinaria, que dejen los productos para dejar de fumar (Tabla 9).

\section{Discusión}

Los resultados mostraron una prevalencia de vida de consumo de cigarrillo de $68.68 \%$, mayor a lo observado en estudiantes de medicina de India donde encontraron que el $10.9 \%$ habían probado el cigarrillo (12). La edad de inicio fue de 16 años, un poco por encima del promedio mundial reportado en la población general que es de 13 a 15 años (13), pero dentro del promedio nacional que es de 12 a 17 años en población general $(14,15)$ y no distante de lo encontrado en el personal de salud de Umbría, Italia, donde la edad de inicio era a los 18 años (16), pero muy por debajo de estudios en personal médico como el de Laos, donde el promedio de edad de inicio fue a los 25 años (17) y en el estudio de médicos y enfermeras de Turquía donde la edad de inicio fue a los 21.7 años y los 20.6 años respectivamente (18). Por otra parte, llama la atención el alto consumo en cantidad de cigarrillos o cajetillas de los 44 médicos actualmente fumadores, el $47.73 \%$ fuman un promedio de 2 a 5 cigarrillos por día, por debajo de lo encontrado en Bosnia y Herzegovina que fue de 16 por día(9) y en Turquía donde fue de 19.2 cigarrillos día (18), pero similar a los hallazgos en Laos donde el $54.4 \%$ había fumado 1 a 5 cigarrillos día (17).

Se encontró que el $24.05 \%$ de los médicos han continuado con el hábito del cigarrillo, resultado menor al encontrado en médicos y personal de salud de Croacia, Italia y Turquía donde la prevalencia estaba en $35.1 \%$, 34.53\% y $34.4 \%$ respectivamente $(16,18,19)$, lo que demuestra una población en consumo levemente inferior de lo encontrado por otras encuestas, sin embargo, este resultado es superior al hallado en personal de la salud en Cataluña donde el $16.4 \%$ eran fumadores (20) y en Texas, donde el $9 \%$ de los encuestados eran fumadores, de estos solo el 3\% eran médicos(21). De los fumadores actuales, solo el $12.54 \%$ ha intentado dejar de fumar, resultado bajo comparado con el obtenido en la implementación de la GHPSS donde $60 \%$ de los estudiantes deseaban cesar el hábito(22) y al encontrado en personal de la salud en Chipre donde el $71.8 \%$ desean abandonar el cigarrillo (23), aunque similar al evidenciado en médicos familiares de Bosnia y Herzegovina donde el $17 \%$ eran exfumadores (9). En cuanto al grado de dependencia al cigarrillo el $20.45 \%$ sienten deseos de fumar a los 60 minutos de despertar, resultado inferior al encontrado en profesionales de la salud en España y en Laos, donde el $54.2 \%$ y el $43 \%$ tiene deseo de fumar a los 60 minutos de despertar(17,24).

La asociación encontrada entre la exposición al hábito de fumar por parte de los padres y el consumo de tabaco en los

Tabla 9. Opinión acerca de la prohibición y los conocimientos

\begin{tabular}{lrr}
\hline \multicolumn{1}{c}{ Opinión } & & \multicolumn{1}{c}{ No } \\
\hline $\begin{array}{l}\text { ¿La venta de cigarrillos a adolescentes (personas menores de 18 años) debe ser } \\
\text { prohibida? }\end{array}$ & $428(99.3 \%)$ & $3(0.7 \%)$ \\
$\begin{array}{l}\text { ¿Debe haber una prohibición total a la publicidad de productos de cigarrillo? } \\
\text { ¿Está de acuerdo con la prohibición de fumar en restaurantes? }\end{array}$ & $380(88.58 \%)$ & $49(11.42 \%)$ \\
¿Está de acuerdo con la prohibición de fumar en discotecas, bares y cafeterías? & $427(99.97 \%)$ & $4(0.93 \%)$ \\
¿Está de acuerdo con la prohibición de fumar en lugares públicos? & $412(95.59 \%)$ & $19(4.41 \%)$ \\
¿Los profesionales de la salud que fuman menos, son los que probablemente & $407(94.43 \%)$ & $24(5.57 \%)$ \\
$\begin{array}{l}\text { aconsejan a los pacientes dejar de fumar? } \\
\text { ¿Cree usted que las posibilidades de que un paciente deje de fumar aumentan si un } \\
\text { profesional de la salud se lo aconseja? }\end{array}$ & $312(72.39 \%)$ & $118(27.38 \%)$ \\
$\begin{array}{l}\text { ¿Los profesionales de la salud deben recibir capacitación específica sobre técnicas } \\
\text { para dejar de fumar? }\end{array}$ & $336(77.96 \%)$ & $93(21.58 \%)$ \\
¿Usted cree que los profesionales de la salud ejercen un rol de modelo para los & $424(98.38 \%)$ & $7(1.62 \%)$ \\
pacientes y el público? & & \\
¿Los profesionales de la salud deben recomendar a los pacientes de forma rutinaria & $372(86.51 \%)$ & $58(13.49 \%)$ \\
los métodos para dejar de fumar? & $390(90.49 \%)$ & $41(9.51 \%)$ \\
¿Los profesionales de la salud deben recomendar a los pacientes de forma rutinaria, & $149(34.57 \%)$ & $279(64.73 \%)$ \\
$\begin{array}{l}\text { que dejen los productos para dejar de fumar? } \\
\text { pacientes acerca de cómo dejar de fumar? }\end{array}$ & $413(95.82 \%)$ & $18(4.18 \%)$ \\
\hline
\end{tabular}


médicos, muestra la importancia de esta variable, aunque se requieren más estudios ya que hasta el momento no se encuentra literatura que evalúe esta asociación en médicos. El 91.42\% reportó no haber estado expuestos en los 7 días anteriores al diligenciamiento de la encueta al humo de segunda mano en la vivienda, lo cual difiere de los datos encontrados en la implementación del GHPSS en Albania y Cuba donde el $71.3 \%$ y el $72.5 \%$ respectivamente reportaron exposición en el hogar (22); sin embargo, el $55.16 \%$ ha estado expuesto en diferentes sitios por lo menos a una persona en los últimos 7 días, cercano a lo reportado en la GHPSS donde más del $70 \%$ de los estudiantes encuestados reportó exposición al humo de segunda mano en ese periodo de tiempo(22).

Las medidas como la prohibición de fumar en lugares públicos ha disminuido de manera considerable la característica de fumador social; sin embargo, en este estudio el $44.62 \%$ consumen cigarrillos en eventos públicos, similar a lo encontrado en enfermeras en Iquique (Chile) donde el $36 \%$ lo hacen en esos escenarios(25).

En cuanto a conocimiento sobre normatividad vigente, el $75.64 \%$ de los encuestados saben que existe una política de restricción de consumo en espacios públicos cerrados, similar al estudio de Laos en el que el $80.8 \%$ conoce acerca de la prohibición de consumo en el sitio de trabajo(17) y en Perugia donde el $99.16 \%$ afirma conocer la prohibición de fumar en lugares públicos (16). Lo anterior, es consistente con el estudio realizado en España con estudiantes de medicina y enfermería, en el que el $97.9 \%$ de los participantes conocen y están de acuerdo con la prohibición de fumar en lugares cerrados (26); aunque algo diferente al reporte de la implementación de la GHPSS donde este conocimiento fue cercano $60 \%$ en 21 de los 48 países encuestados; mientras que en estudiantes de medicina de la India donde el $48 \%$ reconoce dicha política $(22,27)$. Sin embargo, el $8.14 \%$ no tienen claridad si para el lugar de trabajo también existe una política específica. En cuanto a la percepción sobre el cumplimiento de la política, el 6.28\% afirma que no se cumple en su lugar de trabajo, mientras que $45.30 \%$ de los médicos de Laos aseguran que si se les permiten fumar en el trabajo(17).

En la evaluación de los conocimientos del médico para asesorar al paciente revela la importancia que ven ante el tema y que no todos cuentan con la preparación completa; $48.84 \%$ aseguran conocer las terapias, pero solo el $64.72 \%$ sabe de la asesoría integral sobre el consumo de tabaquismo, resultado similar al encontrado en Colombia en los estudiantes de medicina; allí se reportó que el 58.3\% de los estudiantes de quinto año sentían que tenían el suficiente conocimiento para asesorar a sus pacientes, mientras que en Laos, tres cuartas partes de los encuestados no habían recibido ningún tipo de educación completa en el tema(10, 17) y en los médicos del Reino Unido el $90 \%$ siente que no tiene la preparación para asesorar a sus pacientes (28). Lo anterior es de gran importancia, ya que se ha demostrado que los médicos que recibieron educación médica continua en tabaquismo tiene 1.5 a 2.5 más probabilidades de informar sobre los efectos y hacer seguimiento a los pacientes $(\mathrm{p}<0.05)(29)$; además, se ha reportado que los médicos y enfermeras que tienen el conocimiento necesario para asesorar sobre cómo abandonar el hábito de fumar son aquellos que reportaron aconsejar más a sus pacientes $(92 \%)$ (23).

En este estudio el $99.30 \%$ apoya las políticas de prohibición a la venta y el $88.58 \%$ la disminución de la publicidad del tabaco, similar a lo encontrado en Bosnia y Herzegovina y en dos estudios en la India donde el $93.2 \%$ y el $91.6 \%$ respectivamente está de acuerdo con la prohibición de la venta y la publicidad $(9,12,30)$ pero, diferente a lo encontrado en Laos donde los fumadores eran menos propensos a apoyar la prohibición de la publicidad y los avisos en los paquetes de cigarrillos $(\mathrm{p}<0.01)(17)$.

En cuanto a la evaluación del rol del médico ante los pacientes y el público el $86.51 \%$ de los encuestados creen que sí ejercen un modelo a seguir y un papel en el manejo del cese del cigarrillo, similar a lo observado en diferentes estudios en la India y Turquía donde entre el 90\% y el 95\% los ven como un rol a seguir $(12,18,22,27)$; sin embargo, puede ser muy variable. Varios estudios recientes demuestran que el personal de la salud no se ve a sí mismo como un ejemplo, mostrando diferentes porcentajes que oscilan entre $44.3 \%$ y el $57.89 \%(16,19,31)$; mientras que en la implementación del GHPSS se reportó que en países como Cuba, Guatemala y Panamá el 100\% de los encuestados está de acuerdo con el rol médico de aconsejar -(3234); por su parte, en Laos el $94.9 \%$ de los médicos fumadores y el $97.7 \%$ de los exfumadores creen que las posibilidades de que un paciente cese el hábito aumentan si el médico lo aconseja(17).

Finalmente, se encontró que el $98.38 \%$ considera que se debe realizar educación médica continua en tabaquismo, similar a los hallazgos en otras encuestas que evalúan la necesidad de capacitación por parte del personal de salud y que revelan que más del $90 \%$ están de acuerdo con la implementación de este tipo de temas académicos $(17,22$, $35,36)$; sin embargo, en Kirguistan solo el $63 \%$ de los médicos piensa que se requiere más información (37); lo cual se encuentra sustentado en que el entrenamiento a los profesionales de la salud aumentaba la asesoría con la estrategia de las 5As, sobre todo en brindar ayuda, donde el $79.1 \%$ de los médicos familiares que tenía entrenamiento en tabaquismo realizaba asesoría a los padres de niños con enfermedades respiratorias y cerca $85 \%$ de 1 los profesionales de la salud a nivel de urgencias ofrecían ayuda y asesoría $(29,38)$, cifra que concuerda con el hallazgo de Kats et al., que solo el $5.7 \%$ de las enfermeras y el $5.1 \%$ realizaba esta intervención antes del entrenamiento, comparado con los datos posteriores donde el $22.5 \%$ de las enfermeras y el 18.4\% de los médicos realizó la intervención(39). 


\section{Conclusiones}

El presente estudio resalta la importancia de describir los conocimientos, actitudes y prácticas del personal médico frente al tema del tabaquismo, con antecedentes de consumo activo, pasivo o no consumo frente a su relación con los pacientes sobre este tema y su competencia para asesorarlos de manera adecuada, con el fin de proponer estrategias que impacten en el consumo en la población general a través de este importante actor del sistema de salud.

Se destaca la asociación encontrada entre el antecedente de consumo de cigarrillo de uno o ambos padres y su influencia sobre el inicio de consumo entre el personal médico. Impacta el hecho de que un poco más de la mitad de los profesionales encuestados aseguran no tener el conocimiento suficiente para asesorar a estos pacientes.

Por otra parte, se evidenció que existe aún desconocimiento de la normatividad vigente por parte de un pequeño grupo de profesionales y la inclinación mayoritaria del personal médico a apoyar las medidas regulatorias, así como la necesidad de realizar educación médica continua para mejorar la asesoría a los pacientes fumadores.

Por último, se requieren estudios en Colombia para evaluar el impacto de una estrategia de educación médica continuada para mejorar la asesoría sobre pacientes consumidores, así como el fortalecimiento de políticas públicas que apunten a reducir este importante y ya demostrado factor de riesgo de múltiples enfermedades de gran relevancia.

\section{Responsabilidades éticas}

Protección de personas y animales. Los autores declaran que en esta investigación no se han realizado experimentos en seres humanos ni en animales.

Confidencialidad de los datos. Los autores declaran que en este artículo no aparecen datos de pacientes.

Derecho a la privacidad y consentimiento informado. Los autores declaran que en este artículo no aparecen datos de pacientes.

\section{Conflicto de intereses}

1. WHO [Internet]. World Health Organization; 2017 [cited 2017 Sep 14]; Disponible en: http://www.who. int/tobacco/en/.

2. Organización Mundial de la Salud, FCTC. Convenio marco de la OMS para el control del tabaco. 2003;1-37. Disponible en: http://apps.who.int/iris/ bitstream/10665/42813/1/9243591010.pdf\%5Cnhttp:// www.scielosp.org/scielo.php?script=sci_arttext\&pid=S1
$135-57272003000400005 \&$ Ing = e s \& n rm = iso\&tlng=es $\% 5 C$ nhttp://www.ops.org.bo/cgi/sys/s1a.xic ?DB=A\&DS=BO2.1\&S2=1\&S6=1\&S8=1\&EI=(TABA\% 2524

3. Social M de salud y proteccion. Ley 1335 de 2009 [Internet]. 2009 p. 11. Disponible en: http://www. alcaldiabogota.gov.co/sisjur/normas/Norma1.jsp?i=368 78

4. Michael Eriksen; Judith Mackay; Neil Schluger; Farhad Islami Gomeshtapeh; Jeffrey Drope. The Tobacco Atlas [Internet]. American Cancer Society, Inc. American Cancer Society, Inc.; 2015. 1-86 p. Disponible en: http://www.tobaccoatlas.org/

5. World Health Organization. Monitoring tobacco use and prevention policies. 2017.1-135 p.

6. Boyle $P$, Yasantha Ariyaratne M, Barrington R, Bartelink $\mathrm{H}$, Bartsch G, Berns A, et al. Tobacco: deadly in any form or disguise. Lancet. 2006; 367(9524): 1710-2.

7. Centers for Disease Control and Prevention. Tobacco Use and Cessation Counseling - Global Health Professionals Survey Pilot Study, services vices [Internet]. Morbidity and Mortality Weekly Report. Atlanta; 2005. Disponible en: https://www.cdc.gov/ mmwr/PDF/wk/mm5420.pdf

8. Tong EK, Strouse R, Hall J, Kovac M, Schroeder SA. National survey of U.S. health professionals' smoking prevalence, cessation practices, and beliefs. Nicotine Tob Res. 2010; 12(7): 724-33.

9. Hodgetts G, Broers T, Godwin M. Smoking behaviour, knowledge and attitudes among Family Medicine physicians and nurses in Bosnia and Herzegovina. BMC Fam Pract [Internet]. 2004; 5:12. Disponible en: http://www.pubmedcentral.nih.gov/articlerender.fcgi?art id $=449709 \&$ tool $=$ mcentrez\&rendertype $=a b s t r a c t$

10. Rodriguez. DRORCCMN. Smoking in Colombian Medical Schools: The Hidden Curriculum. Prev Med (Baltim). 2001; 33: 170-4.

11. World Health Organization. Global Health Professional Students Survey. 2008; 2008(January 2007): 1-9.

12. Boopathirajan R, Muthunarayanan L. Awareness, Attitude and Use of Tobacco among Medical Students in Chennai. J Lifestyle Med [Internet]. 2017; 7(1):27-34 Disponible en: http://www.jlifestylemed.org/ journal/DOlx.php?id=10.15280/jlm.2017.7.1.27

13. Group GYTSC. Tobacco use among youth: a cross country comparison. Tob Control [Internet]. $2002 ; 11(3): 252-70$. Disponible en: http://ovidsp.ovid.com/ovidweb.cgi?T=JS\&PAGE=refer ence \&D $=$ med4\&NEWS $=\mathrm{N} \& A N=12198280$

14. Wiesner C C, Peñaranda D. Encuesta Mundial de Tabaquismo en Jóvenes. Reporte de Bogotá, Colombia. Rev Colomb Cancerol [Internet]. 2002;6(4):5-14. Disponible en: http://www.cancer.gov.co/ documentos/revistas/2002/pub4/2 - Encuesta mundial de tabaquismo en j?venes.pdf

15. Rodriguez, J, Ruiz F, Peñaloza E, Eslava J, Gómez LC, Sánchez H, Amaya JL, Arenas R BY. Encuesta Nacional de Salud 2007. Resultados Nacionales [National Health Survey 2007. National Results] [Internet]. 2009. 27-260 p. Disponible en: https://www.minsalud.gov.co/ salud/Documents/Córdoba.pdf

16. Pianori D, Gili A, Masanotti G. Changing the smoking habit: Prevalence, knowledge and attitudes among Umbrian hospital healthcare professionals. J Prev Med 
Hyg. 2017;58(1): E72-8.

17. Vanphanom S, Morrow M, Phengsavanh A, Hansana V, Phommachanh S, Tomson T. Smoking among Lao medical doctors: challenges and opportunities for tobacco control. Tob Control [Internet]. 2011; 20 ( 2 ): $144-50$. D i s p o n i ble e n : http://tobaccocontrol.bmj.com/cgi/doi/10.1136/tc.2009.0 35196

18. Sonmez Cl, Aydin LY, Turker Y, Baltaci D, Dikici S, Sariguzel YC, et al. Comparison of smoking habits, knowledge, attitudes and tobacco control interventions between primary care physicians and nurses. Tob Induc Dis [Internet]. Tobacco Induced Diseases; 2015; $13(1): 37$ D i s p o n i b l e e n http://www.tobaccoinduceddiseases.com/content/13/1/ 37

19. Juranić B, Rakošec Ž, Jakab J, Mikšić Š, Vuletić S, Ivandić $\mathrm{M}$, et al. Prevalence, habits and personal attitudes towards smoking among health care professionals. J Occup Med Toxicol [Internet]. 2017; 12(1):20. Disponible en: http://occupmed.biomedcentral.com/articles/10.1186/s12995-0170166-5

20. Martínez C, Martínez-Sánchez JM, Antón L, Riccobene A, Fu M, Quirós N, et al. Prevalencia de consumo de tabaco en trabajadores hospitalarios: metaanálisis en 45 hospitales catalanes TT - Smoking prevalence in hospital workers: meta-analysis in 45 Catalan hospitals. Gac Sanit [Internet]. 2016; 30(1):55-8. Disponible en: http://www.scielosp.org/scielo.php?script=sci_arttext\&p id=S0213-91112016000100011\&lang=pt

21. Shahbazi S, Arif AA, Portwood SG, Thompson ME. Risk factors of smoking among health care professionals. J Prim Care Community Health [Internet]. 2014; $5(4): 228-33$. D i s p o n i ble e n : http://www.ncbi.nlm.nih.gov/pubmed/24695770

22. Warren CW, Sinha DN, Lee J, Lea V, Jones NR. Tobacco use, exposure to secondhand smoke, and cessation counseling among medical students: crosscountry data from the Global Health Professions Student Survey (GHPSS), 2005-2008. BMC Public Health [Internet]. BioMed Central Ltd; 2011; 11(1):72. D i s p o n i b l e e n : http://www.pubmedcentral.nih.gov/articlerender.fcgi?art $i d=3042391 \&$ tool $=$ pmcentrez\&rendertype $=$ abstract

23. Zinonos S, Zachariadou T, Zannetos S, Panayiotou AG, Georgiou A. Smoking prevalence and associated risk factors among healthcare professionals in Nicosia general hospital, Cyprus: a cross-sectional study. Tob Induc Dis [Internet]. Tobacco Induced Diseases; 2016; $14(1): 14$ D i s p o n i b l e e n : http://tobaccoinduceddiseases.biomedcentral.com/artic les/10.1186/s12971-016-0079-6

24. Jiménez-Ruiz CA, Miranda JAR, Pinedo AR, Martinez $\mathrm{EDH}$, Marquez FL, Cobos LP, et al. Prevalence of and attitudes towards smoking among Spanish health professionals. Respiration. 2015; 90(6):474-80.

25. Siqués $P$, Brito J, Muñoz C, Pasten P, Zavala P, Vergara $\mathrm{J}$. Prevalence and characteristics of smoking in primary healthcare workers in Iquique, Chile. Public Health. 2006; 120(7):618-23.

26. Martínez C, Méndez C, Sánchez M, Martínez-Sánchez JM. Attitudes of students of a health sciences university towards the extension of smoke-free policies at the university campuses of Barcelona (Spain). Gac Sanit [Internet]. SESPAS; 2016; 31(2):132-8. Disponible: http://dx.doi.org/10.1016/j.gaceta.2016.08.009

27. Pednekar M, Sinha D, Singh G, Warren C, Asma S, Gupta P, et al. Tobacco use and cessation counseling in India-data from the Global Health Professions Students Survey, 2005-09. Indian J Cancer [Internet]. 2012; $49(4): 425$. D i s p o n i b le e n : http://www.indianjcancer.com/text.asp?2012/49/4/425/1 07751

28. Raupach T, Al-Harbi G, McNeill A, Bobak A, McEwen A. Smoking Cessation Education and Training in U.K. Medical Schools: A National Survey. Nicotine Tob Res [Internet]. 2015; 17(3):372-5. Disponible en: https://academic.oup.com/ntr/articlelookup/doi/10.1093/ntr/ntu199

29. Victor JC, Brewster JM, Ferrence R, Ashley MJ, Cohen JE, Selby P. Tobacco-related medical education and physician interventions with parents who smoke: Survey of Canadian family physicians and pediatricians. Can Fam Physician. 2010; 56(2):157-63.

30. Mony $\mathrm{P}$, Vishwanath $\mathrm{N}$, Krishnan S. Tobacco use, attitudes and cessation practices among healthcare workers of a city health department in Southern India. $J$ Fam Med Prim Care [Internet]. 2015; 4(2):261. Disponible en: http://www.jfmpc.com/text.asp?2015/ 4/2/261/154670

31. Prucha M, Fisher S, Mclntosh S, Grable J, Holderness H, Thevenet-Morrison K, et al. Health Care Workers' Knowledge, Attitudes and Practices on Tobacco Use in Economically Disadvantaged Dominican Republic Communities. Int J Environ Res Public Health [Internet]. $2015 ; 12(4): 4060-75$. Disponible en: http://www.mdpi.com/1660-4601/12/4/4060/

32. Organización Panamericana de la Salud. Cuba Havana - Medical Students (3rd Year Students Only) Global Health Professions Student Survey (GHPSS) FACT. 2008.

33. Organización Panamericana de la Salud. Guatemala Medical Students (3rd Year Students Only) Global Health Professions Student Survey (GHPSS). 2008.

34. Organización Panamericana de la Salud. Panama Medical Students (3rd Year Students Only) Global Health Professions Student Survey (GHPSS). 2008.

35. Armstrong GW, Veronese G, George PF, Montroni I, Ugolini G. Assessment of tobacco habits, attitudes, and education among medical students in the United States and Italy: A cross-sectional survey. J Prev Med Public Heal. 2017; 50(3):177-87.

36. Schoj V, Mejia R, Alderete M, Kaplan CP, Peña L, Gregorich SE, et al. Use of Smoking Cessation Interventions by Physicians in Argentina. J Smok Cessat [Internet]. 2016 Sep 21; 11(3):188-97. Disponible en: http://www.journals.cambridge.org/abstract_S1834261 214000243

37. Brimkulov N, Vinnikov D, Dzhilkiadarova Z, Aralbaeva A. Tobacco use among Kyrgyzstan medical students: an 11-year follow-up cross-sectional study. BMC Public Health [Internet]. BMC Public Health; 2017; 17(1):625. Disponible en: http://bmcpublichealth.biomedcentral. com/articles/10.1186/s12889-017-4547-6

38. Walters EL, Reibling ET, Wilber ST, Sullivan AF, Gaeta TJ, Camargo CA, et al. Emergency Department Provider Preferences Related to Clinical Practice Guidelines for 
Tobacco Cessation: A Multicenter Survey. Fu R, editor. Acad Emerg Med [Internet]. 2014 Jul; 21(7):785-93. Disponible en: http://doi.wiley.com/10.1111/acem.12421 39. Katz DA, Vander Weg MW, Holman J, Nugent A, Baker
$\mathrm{L}$, Johnson S, et al. The emergency department action in smoking cessation (EDASC) trial: Impact on delivery of smoking cessation counseling. Acad Emerg Med. 2012; 19(4):409-20. 